\title{
Corrigendum to Phase Transitions on Fractal Lattices with Long-Range Interactions ${ }^{1}$
}

\section{O. Penrose ${ }^{2}$}

The following proof of Theorem 2 should replace the one given in the paper. Indeed, it proves a slightly stronger version of the theorem, with the restriction $N \geqslant n$ removed.

As in the proof of Theorem 1, we can write $\mathbf{x}$ in the form

$$
\mathbf{x}=\sum_{0}^{q} m^{p} \mathbf{x}^{(p)}, \quad \mathbf{x}^{(0)}, \ldots, \mathbf{x}^{(q)} \in A
$$

where $A$ is the generating set defined on p. 72 of the paper and $q$ is some nonnegative integer depending on $\mathbf{x}$.

To prove the right-hand inequality in Theorem 2, which is equivalent to

$$
R_{N}(x) \leqslant K_{2} N^{1 / D}
$$

define $k$ to be the nonnegative integer satisfying

$$
n^{k-1}<N \leqslant n^{k}
$$

and consider the set $Y$ consisting of all points whose position vectors $\mathbf{y}$ have the form

$$
\mathbf{y}=\sum_{0}^{k-1} m^{p} \mathbf{y}^{(p)}+\sum_{k}^{\infty} m^{p} \mathbf{x}^{(p)}, \quad \mathbf{y}^{(0)}, \ldots, \mathbf{y}^{(k-1)} \in A
$$

where $\mathbf{x}^{(p)}$ is defined to be $\mathbf{0}$ for all $p>q$.

By Theorem 1 each choice of the vectors $\mathbf{y}^{(0)}, \ldots, \mathbf{y}^{(k-1)}$ gives a different $\mathbf{y}$, and since there are $n$ ways of choosing each $\mathbf{y}^{(p)}$, the number of points in

\footnotetext{
${ }^{1}$ This paper appeared in J. Stat. Phys. 45:69-88 (1986).

${ }^{2}$ Mathematics Department, Heriot-Watt University, Edinburgh EH14 4AS, Scotland.
} 
$Y$ is $n^{k}$. These points are all members of the fractal lattice $F$, and their Euclidean distances from $\mathbf{x}$ all satisfy

$$
\begin{aligned}
|\mathbf{y}-\mathbf{x}| & =\left|\sum_{0}^{k-1} m^{p}\left(\mathbf{y}^{(p)}-\mathbf{x}^{(p)}\right)\right| \\
& \leqslant\left(1+m+\cdots+m^{k-1}\right) \rho_{\max } \\
& <m^{k} \rho_{\max } /(m-1)
\end{aligned}
$$

where $\rho_{\max }$ is the Euclidean diameter of $A$; so there are at least $n^{k}$ points of $F$ within a distance $m^{k} \rho_{\max } /(m-1)$ of $\mathbf{x}$. It follows, by the definition of $R_{N}(\mathbf{x})$, that

$$
R_{n^{k}}(\mathbf{x}) \leqslant m^{k} \rho_{\max /(m-1)}
$$

From (C3) and the fact that $R_{N}(\mathbf{x})$ increases monotonically with $N$ we have

$$
R_{N}(\mathbf{x}) \leqslant R_{h^{k}}(\mathbf{x})
$$

and from (3.1) and the left-hand inequality in (C3) we have

$$
\frac{m^{k} \rho_{\max }}{m-1}=\frac{n^{(k-1) / D} m \rho_{\max }}{m-1}<\frac{N^{1 / D} m \rho_{\max }}{m-1}
$$

Combining (C6)-(C8) we verify (C2), with $K_{2}=m \rho_{\max } /(m-1)$.

To prove the left-hand inequality in Theorem 2 , which is equivalent to

$$
R_{N}(\mathbf{x}) \geqslant K_{1} N^{1 / D}
$$

we note that for every pair of vectors $\mathbf{x}, \mathbf{y}$ in $F$ the difference $\mathbf{x}-\mathbf{y}$ can be written in the form

$$
\sum_{p \geqslant 0} m^{p}\left(\mathbf{x}^{(p)}-\mathbf{y}^{(p)}\right)
$$

and is therefore a member of a new fractal lattice $F^{*}$ whose generating set $A^{*}$ consists of all distinct vectors of the form $\mathbf{a}-\mathbf{b}$, with $\mathbf{a}$ and $\mathbf{b}$ in $A$. Let $H$ be the set consisting of all points in $F^{*}$ whose Euclidean distance from the origin is less than $\delta$, where $\delta$ is a length to be chosen later [Eq. (C16)], and let $n(H)$ be the number of points in $H$. Assume for the moment that $N>n(H)$, and let $l$ be the nonnegative integer defined by

$$
n(H) n^{l+1}>N \geqslant n(H) n^{l}
$$


Any vector $\mathbf{z}$ belonging to the original fractal lattice $F$ can be written in the form

$$
\mathbf{z}=\sum_{0}^{l-1} m^{p} \mathbf{z}^{(p)}+m^{l} \mathbf{z}^{\prime}
$$

where $\mathbf{z}^{(0)}, \ldots, \mathbf{z}^{(l-1)}$ belong to $A$ and

$$
\mathbf{z}^{\prime}=\sum_{p \geqslant l} m^{p-l} \mathbf{z}^{(p)}
$$

is a new vector in $F$. Decomposing $\mathbf{x}$ in the analogous way, we see that the Euclidean distance between $\mathbf{z}$ and $\mathbf{x}$ satisfies

$$
\begin{aligned}
|\mathbf{z}-\mathbf{x}| & =\left|\sum_{0}^{l} m^{-1} m^{p}\left(\mathbf{z}^{(p)}-\mathbf{x}^{(p)}\right)+m^{l}\left(\mathbf{z}^{\prime}-\mathbf{x}^{\prime}\right)\right| \\
& \geqslant m^{l}\left|\mathbf{z}^{\prime}-\mathbf{x}^{\prime}\right|-\sum_{0}^{l-1} m^{p}\left|\mathbf{z}^{(p)}-\mathbf{x}^{(p)}\right|
\end{aligned}
$$

The vectors $\mathbf{z}$ in $F$ fall into two classes: $F_{1}$, consisting of those vectors for which $\left|\mathbf{z}^{\prime}-\mathbf{x}^{\prime}\right|<\delta$, and $F_{2}$, for which $\left|\mathbf{z}^{\prime}-\mathbf{x}^{\prime}\right| \geqslant \delta$. The set $F_{1}$ comprises at most $n^{l} n(H)$ points, since there are $n$ choices for each of $z^{(0)}, \ldots, z^{(l-1)}$ and at most $n(H)$ for $\mathbf{z}^{\prime}$, since $\mathbf{z}^{\prime}-\mathbf{x}^{\prime}$ is a member of $H$. For points $\mathbf{z}$ in the set $F_{2}$, we have from $(\mathrm{C} 14)$

$$
|\mathbf{z}-\mathbf{x}| \geqslant m^{\prime} \delta-\left(1+m+\cdots+m^{\prime-1}\right) \rho_{\min }
$$

where $\rho_{\min }$ is the least Euclidean distance between points of $A$. If we now choose $\delta$ as

$$
\delta=\left(m+\frac{1}{m-1}\right) \rho_{\min }
$$

then (C15) implies

$$
|\mathbf{z}-\mathbf{x}|>m^{l+1} \rho_{\min }
$$

for all $\mathbf{z}$ in $F_{2}$. Consequently, all the points $\mathbf{z}$ of $F$ for which $|\mathbf{z}-\mathbf{x}| \leqslant$ $m^{l+1} \rho_{\min }$ belong to $F_{1}$, and since $F_{1}$ comprises at most $n^{l} n(H)$ points, there are at most $n^{l} n(H)$ points of $F$ within a distance $m^{l+1} \rho_{\text {min }}$ of $\mathbf{x}$. Thus, it follows from the definition of $R_{N}(\mathbf{x})$ that

$$
R_{n^{\prime} n(H)}(x) \geqslant m^{l+1} \rho_{\min }
$$


and hence, using (C11), (3.1), and the monotonicity of $R_{N}(\mathbf{x})$ as we did at the end of the proof of $(\mathrm{C} 2)$, that

$$
R_{N}(\mathbf{x}) \geqslant[N / n(H)]^{1 / D} \rho_{\min }
$$

The inequality (C19) was derived on the assumption that $N>n(H)$, but since $R_{N}(\mathbf{x}) \geqslant \rho_{\min }$ for all $N$, the inequality $(\mathrm{C} 19)$ also holds when $N \leqslant n(H)$; therefore (C9) holds for all $N$, with $K_{1}=\rho_{\min } /[n(H)]^{1 / D}$. This completes the proof of Theorem 2. 